Royal Perth Hospital, Wellington Street Campus, GPO Box X2213, Perth, Western Australia 6001, Australia. E-mail: Nicholas.Lawn@ health.wa.gov.au).

COMMENT. The use of dextro-amphetamine or methylphenidate in the treatment of ADHD in adolescents and adults carries a risk of psychostimulant abuse. Factors associated with the highest risk of substance use disorders (SUDs) in patients with ADHD include comorbid antisocial personality disorder, bipolar disorder, eating disorder, and dropping out of school (Kollins SH. Curr Med Res Opin 2008;24:134557). Treatment of ADHD with stimulants during childhood may reduce the risk of developing SUDs at a later age (Biederman J et al. Pediatrics 1999;104:e20). Psychostimulant formulations used in ADHD that have a lesser potential for abuse include long-acting stimulants (eg Concerta, Focalin XR, Adderall XR, Vyvanse), and the skin patch (Daytrana). In patients suspected of an increased risk of SUDs, shortacting formulations of methylphenidate or amphetamine that may be crushed and snorted or injected should be avoided.

Seizure as a complication of therapeutic doses of dextro-amphetamine or mixed amphetamine salts (Adderall) used in ADHD is rarely reported. In a letter to the editor, a 9-year-old mentally retarded girl with ADHD-combined type had a generalized seizure requiring resuscitation after a second dose of Adderall $2.5 \mathrm{mg}$ t.i.d. She was subsequently treated with methylphenidate without seizure recurrence. (Thomas S et al. J Am Acad Child Adolesc Psychiatry 2002;41:365). These authors cite an anecdotal report of convulsions with Ritalin (Chamberlain RW. Pediatrics 1974;54:658-659). Physicians' Desk Reference $\left(55^{\text {th }}\right.$ ed. 2001$)$ cautions against the use of CNS stimulants in children with epilepsy or an abnormal EEG. The incidence of seizures was significantly increased in children with ADHD and centrotemporal spikes in the EEG (Hemmer SA et al. Pediatr Neurol 2001;24:99-102). Some subsequent studies have shown that the addition of methylphenidate is safe in children with ADHD and epilepsy when seizures are controlled with antiepileptic medication (Tan M et al. Arch Dis Child 2005;90:57-59). While the addition of short-acting methylphenidate in children with ADHD and epilepsy controlled with AEDs appears to be safe, a recent controlled study of OROS long-acting methylphenidate suggests potential safety concerns requiring further study. The risk of seizure recurrence increased with increasing doses of OROS-MPH (Gonzalez-Heydrich J, Biederman J et al. Epilepsy Behav 2010;18:229-237). Caution is advised in the use of extended release formulations and larger doses of psychostimulants in the treatment of children with ADHD and a susceptibility to seizures.

\title{
ADHD IN CHILDREN WITH BENIGN EPILEPSY AND SIBLINGS
}

The prevalence and characteristics of ADHD in children with benign epilepsy and in their nonepileptic siblings were compared in a prospective study at Carmel Medical Center, Haifa, Israel. Of 40 patients with benign epilepsy, 21 (52.5\%) had generalized seizures, $16(40 \%)$ had partial seizures, 8 (20\%) had absence epilepsy, and $5(12.5 \%)$ benign epilepsy with centrotemporal spikes. Of 28 (70\%) diagnosed with ADHD, 19 had the inattentive type, 1 with hyperactive type, and 8 with the combined type. Of 12 nonepileptic siblings in the control group, only $2(16.7 \%)$ had ADHD $(\mathrm{P}<0.03)$. Children with seizures more resistant and requiring more than 1 AED showed a trend toward an 
increased risk of attention disorder. Children with more epileptiform discharges in their EEGs were more likely to have ADHD. Of patients with normal EEGs, $50 \%$ had ADHD whereas of children with $>6$ interictal spikes or S/W complexes per minute, $92 \%$ had ADHD. Learning disabilities were more common in the epilepsy group (42.5\% vs $25 \%$ in the control group, $\mathrm{P}<0.05$ ). (Bennett-Back O, Keren A, Zelnik N. Attention-deficit hyperactivity disorder in children with benign epilepsy and their siblings. Pediatr Neurol March 2011;44:187-192). (Respond: Dr Zelnik, Department of Pediatrics, Carmel Medical Center, 7 Michal Street, Haifa 34362, Israel. E-mail: zelnik@ netvision.net.il).

COMMENT. The prevalence of ADHD in school-age children with epilepsy is between 20\% (Gross-Tsur et al. 1997) and 60\% (Sherman et al. 2007). The prevalence of epileptiform EEGs in nonepileptic children evaluated for ADHD is $26 \%$, compared to 3.5-6.5\% in healthy children (Kaufmann et al. 2009; Millichap et al. 2010). The present report shows an increased risk of attention disorder in children with AED-resistant seizures and having more frequent epileptiform discharges in the EEG. ADHD is particularly common in benign epilepsy of childhood with centrotemporal spikes and frontal lobe epilepsy. The comorbidity of ADHD and epilepsy may depend on the effects of AEDs, psychosocial aspects of epilepsy, the type and localization of epileptogenic foci, or even common genetic factors.(Kaufmann R et al. 2009).

Frontal/prefrontal lobe growth retardation is reported in children with BCECTS complicated by cognitive impairments and behavioral problems compared with BCECTS patients without neuropsychiatric deficits. (Kanemura $\mathrm{H}$ et al. Brain Dev Feb 2011;33:106-113). Impaired frontal lobe growth is associated with frequent seizures and spike/wave discharges and neuropsychological problems. A small percentage of BCECTS patients may require early control of seizures to prevent frontal lobe growth retardation and associated cognitive and behavioral problems.

\section{DEVELOPMENTAL DISORDERS}

\section{RISK FACTORS FOR AUTISM IN TUBEROUS SCLEROSIS}

The prevalence and risk factors for autism spectrum disorders (ASD) in a cohort of 103 patients with tuberous sclerosis complex (TSC) were determined in a study at the Massachusetts General Hospital, Boston, MA. ASD was diagnosed in $40 \%$ of patients with TSC. Patients with ASD were younger than those without ASD (9.9 vs 16.2 years, $\mathrm{p}<0.001$ ), and had lower IQs (51 vs $81, \mathrm{p}<0.001$ ). Frequency of male gender was not significantly different ( $49 \%$ vs $42 \%$ ) in patients with and without ASD, and skin, renal, cardiac, pulmonary, and ophthalmological manifestations were similar. TSC/ASD patients were less likely to have mutations in the TSC1 gene. They had an earlier onset of seizures $(0.7$ vs 2.9 years, $\mathrm{p}<0.002)$ and more frequent seizures, and a significantly greater amount of interictal epileptiform features, especially in the left temporal lobe ( $82 \%$ vs $61 \%, \mathrm{p}<0.049)$. Infantile spasms occurred in $59 \%$ of TSC with ASD patients and $32 \%$ of TSC without ASD $(\mathrm{p}<0.014)$. On MRI, the regional distribution of tuber burden was unaltered, but patients with TSC2 and ASD had a higher prevalence of cyst-like tubers. (Numis AL, Major P, Montenegro MA, Muzykewicz DA, Pulsifer MB, Thiele EA. Identification of risk factors for autism spectrum disorder in tuberous sclerosis 